\begin{tabular}{|c|l|}
\hline Title & Scattering Theory for the Dirac Equation with a Nonlocal Term \\
\hline Author(s) & Tsutaya, Kimitoshi; Machihara, Shuji \\
\hline Citation & Hokkaido University Preprint Series in Mathematics, 883, 1-14 \\
\hline Issue Date & 2007 \\
\hline DOI & 10.14943/84033 \\
\hline Doc URL & http://hdl.handle.net/2115/69692 \\
\hline Type & bulletin (article) \\
\hline File Information & pre883.pdf \\
\hline
\end{tabular}

Instructions for use 


\title{
Scattering Theory for the Dirac Equation with a Nonlocal Term
}

\author{
Shuji Machihara* and Kimitoshi Tsutaya ${ }^{\dagger}$ \\ *Department of Mathematics \\ Faculty of Education \\ Saitama University \\ 255 Shimo-Okubo, Sakura-ku \\ Saitama City 338-8570, Japan \\ $\dagger$ Department of Mathematics \\ Hokkaido University \\ Sapporo 060-0810, Japan
}

\begin{abstract}
Consider a scattering problem for the Dirac equation with a nonlocal term including the Hartree type. We show the existence of scattering operators for small initial data in the subcritical and critical Sobolev spaces.
\end{abstract}

\section{Introduction}

We consider a scattering problem for the Dirac equation with a nonlocal term

$$
\partial_{t} \psi+\alpha \cdot \nabla \psi+i \beta \psi=\lambda\left[V *|\psi|^{p-1}\right] \psi, \quad(t, x) \in \mathbf{R} \times \mathbf{R}^{n} .
$$

Here $p \geq 3, n \geq 3, \partial_{t}=\partial / \partial t, \nabla=\left(\partial_{1}, \cdots, \partial_{n}\right), \partial_{j}=\partial / \partial x_{j}, j=1, \cdots, n, \lambda \in \mathbf{C}, \alpha \cdot \nabla=$ $\sum_{j=1}^{n} \alpha_{j} \partial_{j}, \quad \alpha_{j}{ }^{\prime} \mathrm{s}, j=1, \cdots, n$ and $\beta$ are $2^{[(n+1) / 2]} \times 2^{[(n+1) / 2]}$ Hermitian matrices satisfying the usual anticommutation relations ${ }^{\ddagger}$. The unknown function $\psi$ is a $2^{[(n+1) / 2]}$-spinor field defined on $\mathbf{R} \times \mathbf{R}^{n}$. The function $V=V(x)$ satisfies $|V(x)| \leq|x|^{-\gamma}$ with $\gamma>0$, and * denotes the convolution in space.

As a special case of (1.1), the Dirac equation of Hartree type, say $p=3$, with the Coulomb potential $V(x)=|x|^{-1}$ is derived from the Maxwell-Dirac equations with zero magnetic field. See Chadam and Glassey [2]. As another case, Dias and Figueira [3] proved that there exist

\footnotetext{
${ }^{\dagger}$ Partially supported by Grant-in-Aid of formation of COE "Mathematics of Nonlinear Structures via Singularities"

${ }_{\ddagger}^{\ddagger} \alpha_{j} \alpha_{k}+\alpha_{k} \alpha_{j}=2 \delta_{j k} I, \alpha_{j} \beta+\beta \alpha_{j}=0, \beta^{2}=I$ for $1 \leq j, k \leq n$.
} 
weak solutions of a special form of the massless Dirac equation with the nonlinear term $\lambda\left(e^{-|x|}|x|^{-\gamma} *|\psi|^{p}\right) \psi$. See also [4].

In this paper we study the scattering problem for (1.1) with small initial data. The scaling argument for the massless Dirac equation, say $\partial_{t} \psi+\alpha \cdot \nabla \psi=\lambda\left(V *|\psi|^{p-1}\right) \psi$ with $V(x)=|x|^{-\gamma}$ gives the value of the critical Sobolev exponent $s_{c}=(\gamma-1+n(p-3) / 2) /(p-1)$. Our aim of this paper is to prove the existence of scattering operators for (1.1) with small initial data in the subcritical Sobolev space $H^{s}$ with $s>s_{c}$ if $p \geq 3$ and in the critical Sobolev space $H^{s_{c}}$ if $p>3$ under some conditions.

The present paper is organized as follows. In Section 2, we give some notation and conditions, and then state our main results. In Section 3, we prove the results. Our basic tool is the Strichartz estimate for the Klein-Gordon equation which was shown by Machihara, Nakanishi and Ozawa [7]. In Section 4, we state and prove similar results for the critical case $s=s_{c}$ and $p>3$. We use the interpolation inequality shown by Escobedo and Vega [5] for the proof.

\section{Notation and Main Results}

We use the standard notation. We denote by $H_{r}^{s}$ and $B_{r}^{s}$ the usual inhomogeneous Sobolev and Besov spaces on $\mathbf{R}^{n}$, respectively. We write $H^{s}=H_{2}^{s}$. For the definitions of these spaces, see, e.g., [1]. For functions defined on space-time, we write $L_{t}^{q} B_{r}^{s}=L_{t}^{q}\left(\mathbf{R} ; B_{r}^{s}\right)$. We use $A \lesssim B$ to denote the estimate $A \leq C B$, where $C$ is a positive constant.

Let $p \geq 3$. We assume that $(\gamma, s)$ satisfy the following condition:

$$
(\mathrm{H} 1)\left\{\begin{array}{l}
s>s_{c}=\frac{\gamma-1}{p-1}+\frac{n(p-3)}{2(p-1)}, \\
s>\frac{\gamma}{n(p-1)}+\frac{1}{2}, \\
\max \left\{\frac{n(p-3)}{2(p-2)}, 2-\frac{n(p-3)}{2}\right\}<\gamma<n .
\end{array}\right.
$$

The set of $(\gamma, s)$ satisfying (H1) is shown in Figure 1. By choosing $0 \leq \theta \leq 1$ depending on 


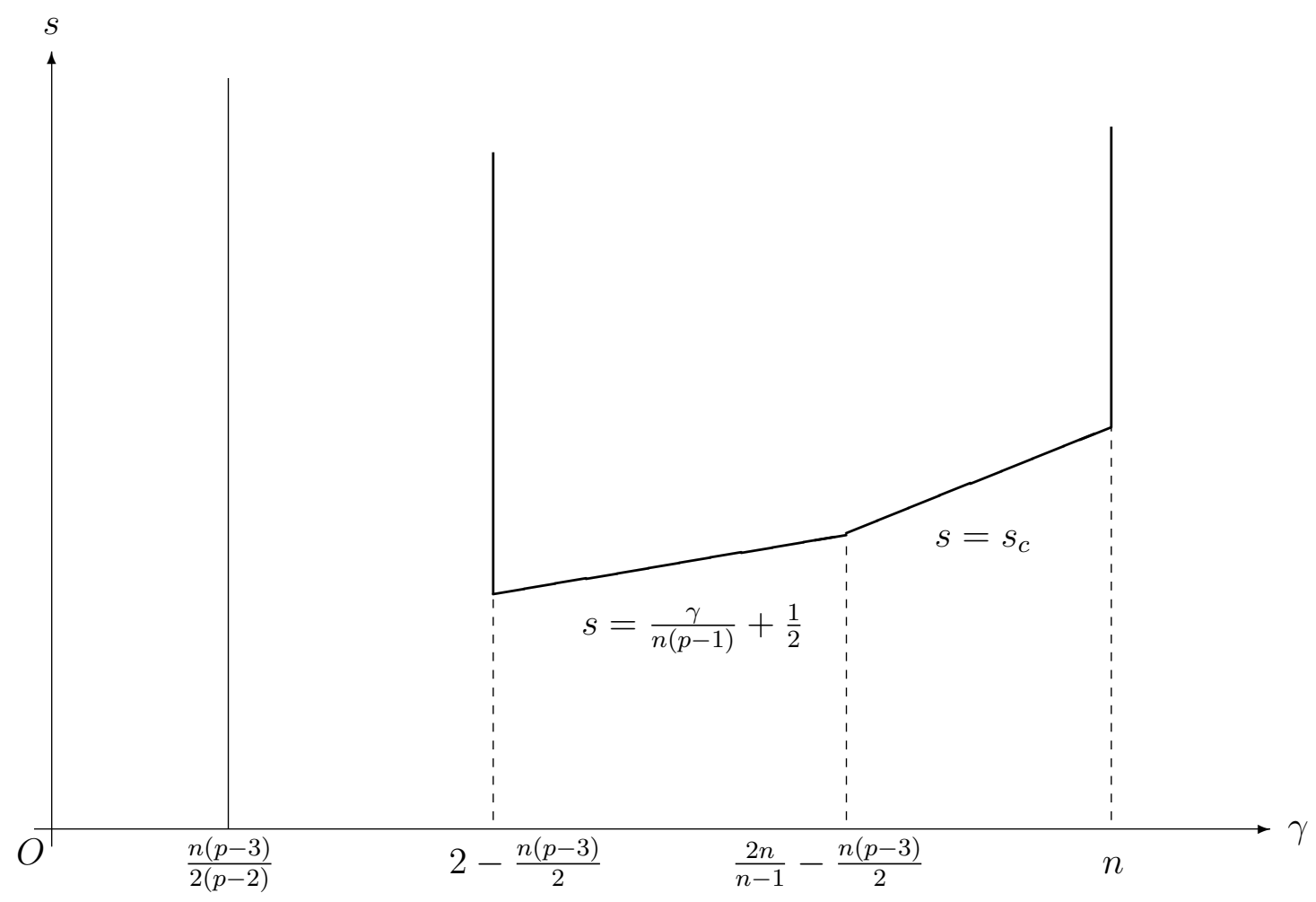

Figure 1: Domain of $(\gamma, s)$ satisfying $(\mathrm{H} 1)$

$s, \gamma, p$ and $n$, we put

$$
\frac{1}{r}=\frac{1}{2}-\frac{2}{(p-1)(n-1+\theta)}, \quad \sigma=\frac{1}{p-1}+\frac{2}{(p-1)(n-1+\theta)} .
$$

We determine $\theta$ in Section 3 so that $s>\sigma$. Note that the pair $(r, \sigma)$ in $(2.1)$ with $p=n=3$ and $\theta=0$ corresponds to the endpoint where the Strichartz estimates fail. See the remark after Lemma 3.1 below. Thus, in case $p=n=3$, we choose $0<\theta \leq 1$.

For $p, r$ and $\sigma$ above and $s>0$, we define $X^{s}$ by

$$
X^{s}=L^{\infty}\left(\mathbf{R} ; H^{s}\right) \bigcap L^{p-1}\left(\mathbf{R} ; B_{r}^{s-\sigma}\right) .
$$

We now state our first main result, which is about the Cauchy problem for (1.1) with the initial data $\psi_{0}(x)$ :

$$
\begin{cases}\partial_{t} \psi+\alpha \cdot \nabla \psi+i \beta \psi=\lambda\left[V *|\psi|^{p-1}\right] \psi, & (t, x) \in \mathbf{R} \times \mathbf{R}^{n}, \\ \psi(0, x)=\psi_{0}(x) & x \in \mathbf{R}^{n} .\end{cases}
$$


Theorem 2.1 (i) Let $p \geq 3$. Assume (H1) and let $s<(p-1) / 2$ if $p$ is not an odd integer. Then there exists $0 \leq \theta \leq 1(0<\theta \leq 1$ if $p=n=3)$, depending only on $s, \gamma, p$ and $n$ such that if $\left\|\psi_{0}\right\|_{H^{s}}$ is sufficiently small, then (2.3) admits a unique global solution $\psi \in X^{s}$ with $s>\sigma$ defined in (2.2).

(ii) For the global solution $\psi$ given in (i), there exist unique $\psi_{ \pm} \in H^{s}$ such that

$$
\lim _{t \rightarrow \pm \infty}\left\|\psi(t)-U(t) \psi_{ \pm}\right\|_{H^{s}}=0
$$

where $U(t)$ denotes the Dirac group, which solves the free Dirac equation.

Remark. (i) The set of $(\gamma, s)$ satisfying (H1) and $s<(p-1) / 2$ is not empty since $p \geq 3$ and $n \geq 3$.

(ii) The condition $s<(p-1) / 2$ in the theorem is not necessary if $p$ is an odd integer. See the proof of Theorem 2.1 in Section 3.

We next consider the final value problem for (1.1) with data given at $t=-\infty$ :

$$
\psi(t, x)=U(t) \psi^{-}(x)+\int_{-\infty}^{t} U\left(t-t^{\prime}\right) F\left(\psi\left(t^{\prime}\right)\right) d t^{\prime},
$$

where $F(\psi)=\lambda\left[V *|\psi|^{p-1}\right] \psi$. Note that $U(t) \psi^{-}(x)$ is a solution of the linear Dirac equation $\partial_{t} \psi+\alpha \cdot \nabla \psi+i \beta \psi=0$ with the initial data $\psi^{-}$at $t=0$.

Theorem 2.2 Let $s, \gamma, p$ and $n$ be as in the preceding theorem. Then there exists $0 \leq \theta \leq 1(0<\theta \leq 1$ if $p=n=3)$, depending only on $s, \gamma, p$ and $n$ such that if $\left\|\psi^{-}\right\|_{H^{s}}$ is sufficiently small, then the itegral equation (2.5) has a unique solution $\psi \in X^{s}$ with $s>\sigma$ satisfying

$$
\lim _{t \rightarrow-\infty}\left\|\psi(t)-U(t) \psi^{-}\right\|_{H^{s}}=0
$$

From Theorems 2.1 and 2.2, we can define the scattering operator for small initial data in the case $p \geq 3$ under the assumptions of Theorem 2.1. See, e.g., [8].

\section{Proofs of Theorems}

To prove Theorem 2.1, we rewrite (2.3) as the following integral equation

$$
\psi(t)=U(t) \psi_{0}+\int_{0}^{t} U\left(t-t^{\prime}\right) F\left(\psi\left(t^{\prime}\right)\right) d t^{\prime}
$$


where $F(\psi)=\lambda\left[V *|\psi|^{p-1}\right] \psi$, and $U(t)$ is the free propagator defined on $L^{2}\left(\mathbf{R}^{n} ; \mathbf{C}^{2^{[(n+1) / 2]}}\right)$ given by

$$
U(t)=I \cos t(1-\Delta)^{1 / 2}-(\alpha \cdot \nabla+i \beta)(1-\Delta)^{-1 / 2} \sin t(1-\Delta)^{1 / 2} .
$$

The following lemma is the Strichartz estimates for $U(t)$. See [7].

Lemma 3.1 Let $n \geq 2$. Then one has the estimates

$$
\begin{array}{r}
\|U(t) u\|_{L_{t}^{q_{1} B_{r_{1}}}-s_{1}} \lesssim\|u\|_{L^{2}}, \\
\left\|\int_{t^{\prime}<t} U\left(t-t^{\prime}\right) F\left(t^{\prime}\right) d t^{\prime}\right\|_{L_{t}^{q_{2} B_{r_{2}}^{-s_{2}}}} \lesssim\|F\|_{L_{t}^{q_{3}^{\prime} B_{r_{3}^{\prime}}^{s_{3}}}},
\end{array}
$$

where $2 / q_{j}=(n-1+\theta)\left(1 / 2-1 / r_{j}\right), 2 s_{j}=(n+1+\theta)\left(1 / 2-1 / r_{j}\right)$ for $0 \leq \theta \leq 1$, $2 \leq q_{j}, r_{j} \leq \infty,\left(q_{j}, r_{j}\right) \neq(2, \infty), j=1,2,3$ when $n=2,3$, and $p^{\prime}$ denotes the conjugate exponent to $p$, i.e., $1 / p+1 / p^{\prime}=1$.

Remark. When we apply Lemma 3.1 in the case $n=3$ and $q=2$, we take $\theta>0$ since $\left(q_{j}, r_{j}\right) \neq(2, \infty)$ which is called "end point" where the Strichartz estimates fail.

Proof of Theorem 2.1. We first prove Part (i) of Theorem 2.1. For $(s, \gamma)$ satisfying (H1), if $n(p-3) /(2(p-2))<2 n /(n-1)-n(p-3) / 2$, we choose $0 \leq \theta \leq 1(0<\theta \leq 1$ if $p=n=3)$ such that

$$
s>\frac{\gamma-1}{p-1}+\frac{n(p-3)}{2(p-1)}+\frac{2 \theta}{(p-1)(n-1+\theta)}
$$

and

$$
\frac{n(p-3)}{2(p-2)} \leq \frac{2 n}{n-1+\theta}-\frac{n(p-3)}{2}<\gamma<n
$$

On the other hand, if $n(p-3) /(2(p-2)) \geq 2 n /(n-1)-n(p-3) / 2$, we take $\theta=0$. In either case, we have by $(\mathrm{H} 1)$

$$
\frac{2 n}{n-1+\theta}-\frac{n(p-3)}{2}<\gamma<n .
$$

Using $\theta$ chosen above, we set (2.1). 
Let $M>0$. We define the complete metric space $X_{M}^{s}$ by

$$
\begin{aligned}
X_{M}^{s} & =\left\{\psi \in X^{s}:\|\psi\|_{X^{s}} \leq M\right\} \\
\|\psi\|_{X^{s}} & =\|\psi\|_{L_{t}^{\infty} H^{s}}+\|\psi\|_{L_{t}^{p-1} B_{r}^{s-\sigma}}
\end{aligned}
$$

where $r$ and $\sigma$ are defined in (2.1).

Let $A$ be the map defined by

$$
A \psi(t)=U(t) \psi_{0}+\int_{0}^{t} U\left(t-t^{\prime}\right) F\left(\psi\left(t^{\prime}\right)\right) d t^{\prime} \quad \text { for } \psi \in X_{M}^{s}
$$

We show that $A$ is a contraction of $X_{M}^{s}$ into itself. From (3.2), (3.3) and (3.7), we have

$$
\|A \psi\|_{X^{s}} \lesssim\left\|\psi_{0}\right\|_{H^{s}}+\|F(\psi)\|_{L_{t}^{1} H^{s}}
$$

Take

$$
\frac{1}{p_{2}}=\frac{1}{\widetilde{q_{2}}}=\frac{1}{p-1}\left(1-\frac{\gamma}{n}\right)
$$

and set

$$
\frac{1}{p_{1}}=\frac{1}{2}-\frac{1}{p_{2}} \text { and } \quad \frac{1}{\widetilde{q_{1}}}=\frac{p-2}{\widetilde{q_{2}}}+\frac{1}{2}
$$

Then (3.9) and (3.10) imply

$$
\frac{1}{p_{1}}=\frac{\gamma}{n}+\frac{1}{\widetilde{q_{1}}}-1
$$

We note that $0<1 / p_{1}<1 / 2$ and $1 / 2<1 / \widetilde{q_{1}}<1$ since $p \geq 3$ and $\gamma>n(p-3) /(2(p-2))$ by assumption.

We estimate the norm of $F(\psi)=\lambda\left(V *|\psi|^{p-1}\right) \psi$ in (3.8). The following inequality holds:

$$
\begin{aligned}
\|F(\psi)\|_{L_{t}^{1} H^{s}} & \lesssim\left\|V *|\psi|^{p-1}\right\|_{L_{t}^{(p-1) /(p-2)} H_{p_{1}}^{s}}\|\psi\|_{L_{t}^{p-1} L^{p_{2}}}+\left\|V *|\psi|^{p-1}\right\|_{L_{t}^{1} L^{\infty}}\|\psi\|_{L_{t}^{\infty} H^{s}} \\
& \equiv I_{1}+I_{2},
\end{aligned}
$$

where $p_{1}$ and $p_{2}$ are given in (3.9) and (3.10).

We first estimate $I_{1}$. By (3.10) and (3.11), the Hardy-Littlewood-Sobolev and Hölder inequalities yield

$$
\begin{aligned}
\left\|V *|\psi|^{p-1}\right\|_{H_{p_{1}}^{s}} & \lesssim\left\||\psi|^{p-1}\right\|_{H_{\widetilde{q_{1}}}^{s}} \\
& \lesssim\|\psi\|_{L^{\widetilde{q_{2}}}}^{p-2}\|\psi\|_{H^{s}},
\end{aligned}
$$


where $\widetilde{q_{1}}$ and $\widetilde{q_{2}}$ are given in (3.9) and (3.10). We note by (2.1), (3.4) and (3.6) that

$$
\frac{1}{r}-\frac{s-\sigma}{n}<\frac{1}{p-1}\left(1-\frac{\gamma}{n}\right)=\frac{1}{p_{2}}=\frac{1}{\widetilde{q_{2}}}<\frac{1}{r}
$$

Using the embedding $B_{r}^{s-\sigma} \hookrightarrow L^{p_{2}}$ and $B_{r}^{s-\sigma} \hookrightarrow L^{\widetilde{q_{2}}}$, we obtain

$$
I_{1} \lesssim\|\psi\|_{L_{t}^{p-1} B_{r}^{s-\sigma}}^{p-1}\|\psi\|_{L_{t}^{\infty} H^{s}}
$$

We next estimate $I_{2}$. We use the following proposition:

Proposition 3.2 Let $p \geq 3$. Let $\gamma$ and $s$ satisfy (H1) and let $r$ and $\sigma$ be given in (2.1). Then there exists $p_{5} \geq 1$ such that

$$
\begin{array}{ll} 
& -\frac{\gamma}{n}-\frac{1}{r}+1<\frac{p-2}{p_{5}}<1-\frac{1}{r}, \\
& 0<\frac{p-2}{p_{5}}<\frac{s-\sigma}{n}-\frac{\gamma}{n}-\frac{1}{r}+1, \\
\text { and } \quad & \frac{1}{r}-\frac{s-\sigma}{n} \leq \frac{1}{p_{5}} \leq \frac{1}{r} .
\end{array}
$$

Proof. It is sufficient to show that

$$
\begin{aligned}
& \frac{s-\sigma}{n}-\frac{\gamma}{n}-\frac{1}{r}+1>0, \\
& m>-\frac{\gamma}{n}-\frac{1}{r}+1, \\
& m>(p-2)\left(\frac{1}{r}-\frac{s-\sigma}{n}\right), \\
& (p-2)\left(\frac{1}{r}-\frac{s-\sigma}{n}\right)<\frac{s-\sigma}{n}-\frac{\gamma}{n}-\frac{1}{r}+1,
\end{aligned}
$$

where $m=\min \{1-1 / r,(p-2) / r\}$. Recall that (3.4) and (3.6) are satisfied. We notice (3.16) is equivalent to (3.6) when $m=(p-2) / r$. If $m=1-1 / r,(3.16)$ holds true since $\gamma>0$. We can also easily verify that (3.18) is equivalent to (3.4) by substituting (2.1) into the both sides of (3.18). Moreover, we see that (3.18) yields (3.15) by $\gamma<n$.

Finally, we show (3.17) dividing into two cases. If $n(p-3) /(2(p-2))<2 n /(n-1)-$ $n(p-3) / 2$, then $m=(p-2) / r$. In fact, from (3.5) we have

$$
\frac{(p-3)(p-1)}{4(p-2)} \leq \frac{1}{n-1+\theta}
$$


which yields

$$
p \leq 3+\frac{(p-3)(p-1)}{p-2} \leq 3+\frac{4}{n-1+\theta} .
$$

We have used $p \geq 3$ for the first inequality. Hence, $m=(p-2) / r$. Clearly, if $m=(p-2) / r$, (3.17) holds true since $s>\sigma$ and $p \geq 3$.

If $n(p-3) /(2(p-2)) \geq 2 n /(n-1)-n(p-3) / 2$, recall $\theta=0$. We have only to deal with the case $m=1-1 / r$ since in the other case (3.17) is clearly true as above. From (H1), we have

$$
\begin{aligned}
s & >s_{c}=\frac{\gamma-1}{p-1}+\frac{n(p-3)}{2(p-1)} \\
& >\frac{1}{p-1}\left(\frac{n(p-3)}{2(p-2)}-1\right)+\frac{n(p-3)}{2(p-1)} \\
& >\frac{1}{p-1}+\frac{2}{(n-1)(p-1)}+\frac{n(p-1)}{p-2}\left(\frac{1}{2}-\frac{2}{(n-1)(p-1)}\right)-\frac{n}{p-2} \\
& =\sigma+\frac{n}{p-2}\left(\frac{p-1}{r}-1\right)
\end{aligned}
$$

since $\theta=0$. By simple calculation we see that the last inequality is equivalent to (3.17) with $m=1-1 / r$. This completes the proof of the proposition.

We now return to the proof of Theorem 2.1. Let $p_{5}$ be as in Proposition 3.2. We set

$$
\frac{1}{p_{3}}=\frac{\gamma}{n}+\frac{1}{p_{4}}-1, \quad \text { and } \quad \frac{1}{p_{4}}=\frac{1}{r}+\frac{p-2}{p_{5}}
$$

for $p \geq 3$. Then by $\gamma<n$ and Proposition 3.2, we see that $0<1 / p_{3}, 1 / p_{4}<1$ and

$$
s-\sigma>\frac{n}{p_{3}} .
$$

By the Sobolev and Hardy-Littlewood-Sobolev inequalities, we have

$$
\left\|V *|\psi|^{p-1}(t)\right\|_{L^{\infty}} \lesssim\left\|V *|\psi|^{p-1}(t)\right\|_{H_{p_{3}}^{s-\sigma}} \lesssim\left\||\psi|^{p-1}\right\|_{H_{p_{4}}^{s-\sigma}}
$$

By the Hölder and Sobolev inequalities and Proposition 3.2, we obtain

$$
\left\||\psi|^{p-1}\right\|_{H_{p_{4}}^{s-\sigma}} \lesssim\|\psi\|_{B_{r}^{s-\sigma}}\|\psi\|_{L^{p_{5}}}^{p-2} \lesssim\|\psi\|_{B_{r}^{s-\sigma}}^{p-1}
$$

and hence,

$$
I_{2} \lesssim\|\psi\|_{L_{t}^{p-1} B_{r}^{s-\sigma}}^{p-1}\|\psi\|_{L_{t}^{\infty} H^{s}}
$$


Combining (3.8), (3.12), (3.14) and (3.19) together, we obtain

$$
\|A \psi\|_{X^{s}} \leq C_{1}\left\|\psi_{0}\right\|_{H^{s}}+C_{2}\|\psi\|_{X^{s}}^{p} \leq C_{1}\left\|\psi_{0}\right\|_{H^{s}}+C_{2} M^{p}
$$

Similarly, we have

$$
\begin{aligned}
\left\|F\left(\psi_{1}\right)-F\left(\psi_{2}\right)\right\|_{L_{t}^{1} H^{s}} & \lesssim\left(\left\|\psi_{1}\right\|_{X^{s}}^{p-1}+\left\|\psi_{2}\right\|_{X^{s}}^{p-1}\right)\left\|\psi_{1}-\psi_{2}\right\|_{X^{s}} \\
& \lesssim M^{p-1}\left\|\psi_{1}-\psi_{2}\right\|_{X^{s}}
\end{aligned}
$$

and hence

$$
\left\|A \psi_{1}-A \psi_{2}\right\|_{X^{s}} \leq C_{3} M^{p-1}\left\|\psi_{1}-\psi_{2}\right\|_{X^{s}}
$$

Thus, if we choose the $\left\|\psi_{0}\right\|_{H^{s}}$ sufficiently small and $M>0$ so that,

$$
\left\|\psi_{0}\right\|_{H^{s}} \leq \frac{M}{2 C_{1}} \text { and } M^{p-1} \leq \min \left\{\frac{1}{2 C_{2}}, \frac{1}{2 C_{3}}\right\}
$$

then we obtain

$$
\|A \psi\|_{X^{s}} \leq M \text { and }\left\|A \psi_{1}-A \psi_{2}\right\|_{X^{s}} \leq \frac{1}{2}\left\|\psi_{1}-\psi_{2}\right\|_{X^{s}}
$$

which shows that $A$ is a contraction of $X_{M}^{s}$ into itself. Therefore, $A$ admits a unique fixed point $\psi \in X^{s}$ which satisfies (3.1).

(ii) Using the global solution $\psi$ given in (i), we define the functions $\psi_{ \pm}$by

$$
\psi_{ \pm}(x)=\psi_{0}(x)+\int_{0}^{ \pm \infty} U\left(-t^{\prime}\right) F\left(\psi\left(t^{\prime}\right)\right) d t^{\prime}
$$

Then $U(t) \psi_{ \pm}$are solutions of the linear Dirac equation $\partial_{t} \psi+\alpha \cdot \nabla \psi+i \beta \psi=0$. Moreover, we see that $\psi_{ \pm} \in H^{s}$ and (2.4) hold since $\|F(\psi)\|_{H^{s}} \in L_{t}^{1}$ by (3.12), (3.14) and (3.19).

This completes the proof of Theorem 2.1.

Proof of Theorem 2.2 We can prove Theorem 2.2 in the same way as the proof of Theorem 2.1. We omit the details.

\section{Critical Case}

In this section, we consider the same problem as in the previous section for the Sobolev exponent including the critical one. Let $p>3$. We make some preparations to state the 
result. We assume that $(\gamma, s)$ satisfy the following conditions:

$$
\left\{\begin{array}{l}
s \geq s_{c}=\frac{\gamma-1}{p-1}+\frac{n(p-3)}{2(p-1)}, \\
\max \left\{\frac{n(p-3)}{2(p-2)}, \frac{2 n}{n-1}-\frac{n(p-3)}{2}\right\}<\gamma<n .
\end{array}\right.
$$

We define some function spaces. We put

$$
\left\{\begin{aligned}
\frac{1}{r_{0}} & =\frac{1}{2}-\frac{2}{(n-1)(p-1)}, \\
\sigma_{0} & =\frac{n+1}{(n-1)(p-1)} .
\end{aligned}\right.
$$

Note that $0<1 / r_{0}<1 / 2$. For any $(\gamma, s)$ satisfying (H2), we have $s>\sigma_{0}$, hence we can choose $\sigma_{1}$ and $\sigma_{2}$ such that

$$
0<\sigma_{2}<\sigma_{0}<\sigma_{1}<\min \left\{(s+1) \sigma_{0} /\left(1+\sigma_{0}\right),(n+1-\gamma) \sigma_{0},(n+1) /(2(n-1))\right\}
$$

For each $\sigma_{j}, j=1,2$, we define $q_{j}$ and $r_{j}, j=1,2$ by

$$
\frac{1}{q_{j}}=\frac{(n-1) \sigma_{j}}{n+1}=\frac{n-1}{2}\left(\frac{1}{2}-\frac{1}{r_{j}}\right), j=1,2 .
$$

Then we see that

$$
2<q_{1}<p-1<q_{2}
$$

For $p, r_{i}, \sigma_{i}, i=0,1,2, q_{j}, j=1,2$ above and $s>0$, we set

$$
Y^{s}=L^{\infty}\left(\mathbf{R} ; H^{s}\right) \bigcap L^{p-1}\left(\mathbf{R} ; B_{r_{0}}^{s-\sigma_{0}}\right) \bigcap L^{q_{1}}\left(\mathbf{R} ; B_{r_{1}}^{s-\sigma_{1}}\right) \bigcap L^{q_{2}}\left(\mathbf{R} ; B_{r_{2}}^{s-\sigma_{2}}\right) .
$$

Theorem 4.1 Let $p>3$ and let $(\gamma, s)$ satisfy (H2). Assume that $s<(p-1) / 2$ if $p$ is not an odd integer. If $\left\|\psi_{0}\right\|_{H^{s}}$ is sufficiently small, then there exists a unique global solution $\psi$ of (2.3) such that $\psi \in Y^{s}$ defined in (4.5).

Moreover, for the global solution $\psi$ given in (i), there exist unique $\psi_{ \pm} \in H^{s}$ satisfying (2.4).

Theorem 4.2 Let $s, \gamma, p$ and $n$ be as in the preceding theorem. If $\left\|\psi^{-}\right\|_{H^{s}}$ is suffciently small, then the integral equation (2.5) has a unique solution $\psi \in Y^{s}$ satisfying (2.6). 
From Theorems 4.1 and 4.2, we can also define the scattering operator for small initial data in the case $p>3$ under the assumptions of Theorem 4.1.

Proof of Theorem 4.1. For $M>0$, we define the complete metric space $Y_{M}^{s}$ by

$$
\begin{aligned}
Y_{M}^{s} & =\left\{\psi \in Y^{s}:\|\psi\|_{Y^{s}} \leq M\right\}, \\
\|\psi\|_{Y^{s}} & =\|\psi\|_{L_{t}^{\infty} H^{s}}+\|\psi\|_{L_{t}^{p-1} B_{r_{0}}^{s-\sigma_{0}}}+\|\psi\|_{L_{t}^{q_{1}} B_{r_{1}}^{s-\sigma_{1}}}+\|\psi\|_{L_{t}^{q_{2}} B_{r_{2}}^{s-\sigma_{2}}},
\end{aligned}
$$

where $r_{i}, \sigma_{i}, i=0,1,2$ and $q_{j}, j=1,2$ are defined in (4.1)-(4.3).

We proceed in a similar way to the proof of Theorem 2.1 to show that the map $A$ defined in (3.7) is a contraction of $Y_{M}^{s}$ into itself. As before, we have

$$
\|A \psi\|_{Y^{s}} \lesssim\left\|\psi_{0}\right\|_{H^{s}}+\|F(\psi)\|_{L_{t}^{1} H^{s}}
$$

We choose $p_{1}, p_{2}, \widetilde{q_{1}}$ and $\widetilde{q_{2}}$ as in (3.9) and (3.10). Then we have (3.12). We can estimate $I_{1}$ in the same manner as before. However, as the Sobolev embedding for $\|\psi\|_{L^{\widetilde{q 2}}}$ in (3.13) and $\|\psi\|_{L^{p_{2}}}$ in $I_{1}$, we use

$$
B_{r_{0}}^{s-\sigma_{0}} \hookrightarrow B_{r_{0}}^{s_{c}-\sigma_{0}} \hookrightarrow L^{p_{2}}, L^{\widetilde{q_{2}}}
$$

In fact, (3.9) and (4.1) imply

$$
\frac{1}{r_{0}}-\frac{s_{c}-\sigma_{0}}{n}=\frac{1}{p-1}\left(1-\frac{\gamma}{n}\right)=\frac{1}{p_{2}}=\frac{1}{\widetilde{q_{2}}},
$$

and $s \geq s_{c}$ by assumption. Thus, we obtain

$$
I_{1} \lesssim\|\psi\|_{L_{t}^{p-1} B_{r_{0}}^{s-\sigma_{0}}}^{p-1}\|\psi\|_{L_{t}^{\infty} H^{s}}
$$

We next estimate $I_{2}$ following Escobedo and Vega [5]. Since $q_{1}$ and $q_{2}$ given in (4.3) satisfy (4.4), there exists $0<\delta<1$ such that

$$
\delta\left(1-\frac{p-1}{q_{1}}\right)+(1-\delta)\left(1-\frac{p-1}{q_{2}}\right)=0 .
$$

We show that

$$
\left\|V *|\psi|^{p-1}\right\|_{L_{t}^{1} L^{\infty}} \lesssim\|\psi\|_{L_{t}^{q_{1} B_{r_{1}}^{s-\sigma_{1}}}}^{(p-1) \delta}\|\psi\|_{L_{t}^{q_{2}} B_{r_{2}}^{s-\sigma_{2}}}^{(p-1)(1-\delta)},
$$

where $r_{j}$ and $\sigma_{j}, j=1,2$ satisfy (4.2) and (4.3). We use the following lemma: 
Lemma 4.3 Let $1<a, b<\infty, 0<\alpha, \beta<n$ and $0<\delta<1$ satisfy

$$
\begin{aligned}
& \delta\left(\frac{1}{a}-\frac{\alpha}{n}\right)+(1-\delta)\left(\frac{1}{b}-\frac{\beta}{n}\right)=0, \\
& \frac{1}{a}-\frac{\alpha}{n} \neq 0 \quad \text { and } \quad \frac{1}{b}-\frac{\beta}{n} \neq 0 .
\end{aligned}
$$

Then we have

$$
\|u\|_{L^{\infty}\left(\mathbf{R}^{n}\right)} \lesssim\|u\|_{H_{a}^{\alpha}}^{\delta}\|u\|_{H_{b}^{\beta}}^{1-\delta}
$$

See [5] for its proof.

By (4.2), we have

$$
\left(\sigma_{1}<\right) \sigma_{1}\left(\frac{1}{\sigma_{0}}+1\right)-1<s
$$

which is equivalent to

$$
\frac{p-1}{q_{1}}-1<s-\sigma_{1}
$$

since $\sigma_{0}=(n+1) /((n-1)(p-1))$ and $1 / q_{1}=(n-1) \sigma_{1} /(n+1)$ by $(4.3)$. Hence, we can take $\alpha>0$ such that

$$
0<\frac{p-1}{q_{1}}-1<\alpha<\min \left\{s-\sigma_{1}, n+\frac{p-1}{q_{1}}-1\right\}
$$

We can also take $\beta>0$ such that

$$
0<\beta<\min \left\{s-\sigma_{2}, n+\frac{p-1}{q_{2}}-1\right\}
$$

by (4.2) and (4.4). Set

$$
\frac{1}{a}=\frac{\alpha}{n}+\frac{1}{n}\left(1-\frac{p-1}{q_{1}}\right) \quad \text { and } \quad \frac{1}{b}=\frac{\beta}{n}+\frac{1}{n}\left(1-\frac{p-1}{q_{2}}\right) .
$$

We see by (4.11) and (4.12) that $0<1 / a<1$ and $0<1 / b<1$. Note that (4.10) is satisfied by (4.8). Applying Lemma 4.3 for $u=V *|\psi|^{p-1}$, we have

$$
\left\|V *|\psi|^{p-1}(t)\right\|_{L^{\infty}} \lesssim\left\|V *|\psi|^{p-1}(t)\right\|_{H_{a}^{\alpha}}^{\delta}\left\|V *|\psi|^{p-1}(t)\right\|_{H_{b}^{\beta}}^{1-\delta}
$$

To estimate the $H_{a}^{\alpha}$ - norm in (4.13), we write

$$
\frac{1}{a}=\frac{\gamma}{n}+\frac{p-2}{a_{1}}+\frac{1}{a_{2}}-1
$$


where

$$
\frac{1}{a_{1}}=\frac{1}{r_{1}}-\frac{s_{c}-\sigma_{1}}{n}, \quad \frac{1}{a_{2}}=\frac{\alpha}{n}+\frac{1}{r_{1}}-\frac{s_{c}-\sigma_{1}}{n}
$$

with $s_{c}=(\gamma-1+n(p-3) / 2) /(p-1)$. We note that

$$
0<\frac{1}{a_{1}}<\frac{1}{a_{2}}<\frac{1}{2}
$$

In fact, we can verify $0<1 / a_{1}<1 / a_{2}$ using (4.15),

$$
\frac{1}{r_{1}}=\frac{1}{2}-\frac{2 \sigma_{1}}{n+1}, \quad \sigma_{0}=\frac{n+1}{(n-1)(p-1)} \text { and } \sigma_{1}<(n+1-\gamma) \sigma_{0}
$$

in (4.1)-(4.3). Moreover, we have $1 / a_{1}<1 / a_{2}<1 / r_{1} \leq 1 / 2$ by (4.11) and (4.15). Note that $s_{c}-\sigma_{1}>0$ holds by assumption and (4.2).

By (4.14), the Hardy-Littlewood-Sobolev and Hölder inequalities give

$$
\left\|V *|\psi|^{p-1}(t)\right\|_{H_{a}^{\alpha}}^{\delta} \lesssim\|\psi(t)\|_{L^{a_{1}}}^{(p-2) \delta}\|\psi(t)\|_{H_{a_{2}}^{\alpha}}^{\delta}
$$

It follows from (4.15), $s \geq s_{c}$ and the embedding $B_{r_{1}}^{s-\sigma_{1}} \hookrightarrow L^{a_{1}}$ and $B_{r_{1}}^{s-\sigma_{1}} \hookrightarrow H_{a_{2}}^{\alpha}$ that

$$
\left\|V *|\psi|^{p-1}(t)\right\|_{H_{a}^{\alpha}}^{\delta} \lesssim\|\psi(t)\|_{B_{r_{1}}^{s-\sigma_{1}}}^{(p-1) \delta}
$$

Similarly, the $H_{b}^{\beta}$ - norm in (4.13) is estimated by

$$
\left\|V *|\psi|^{p-1}(t)\right\|_{H_{b}^{\beta}}^{1-\delta} \lesssim\|\psi(t)\|_{B_{r_{2}}^{s-\sigma_{2}}}^{(p-1)(1-\delta)}
$$

where $r_{2}$ and $\sigma_{2}$ are given by (4.2) and (4.3) with $j=2$. Hence, from (4.13) we have

$$
\left\|V *|\psi|^{p-1}(t)\right\|_{L^{\infty}} \lesssim\|\psi(t)\|_{B_{r_{1}}^{s-\sigma_{1}}}^{(p-1) \delta}\|\psi(t)\|_{B_{r_{2}}^{s-\sigma_{2}}}^{(p-1)(1-\delta)} .
$$

Thus, we obtain (4.9) by (4.8) and the Hölder inequality. Therefore,

$$
I_{2} \lesssim\|\psi\|_{L_{t}^{q_{1} B_{r_{1}}^{s}}}^{(p-1) \delta}\|\psi\|_{L_{t}^{q_{2}} B_{r_{2}}^{s-\sigma_{2}}}^{(p-1)(1-\delta)}\|\psi\|_{L_{t}^{\infty} H^{s}}
$$

holds. Combining (3.12), (4.6), (4.7) and (4.16) together, we obtain

$$
\|A \psi\|_{Y^{s}} \leq C_{1}\left\|\psi_{0}\right\|_{H^{s}}+C_{2}\|\psi\|_{Y^{s}}^{p} \leq C_{1}\left\|\psi_{0}\right\|_{H^{s}}+C_{2} M^{p}
$$

Therefore, proceeding as in the proof of Theorem 2.1, we obtain the desired result.

Proof of Theorem 4.2.

The proof is the same as that of Theorem 4.1. We omit the details. 


\section{References}

[1] J. Bergh and J. Löfström, Interpolation spaces, Springer, Berlin, 1976.

[2] J. M. Chadam and R. T. Glassey, On the Maxwell-Dirac equations with zero magnetic field and their solution in two space dimensions, J. Math. Anal. and Appl. 53 (1976), 495-507.

[3] J. P. Dias and M. Figueira, Solutions faibles du problème de Cauchy pour certaines équations de Dirac non linéaires, Portugal. Math. 46 (1989), 475-484.

[4] J. P. Dias and M. Figueira, On the existence of weak solutions for nonlinear time dependent Dirac equation, Proc. Royal Soc. of Edinburgh 113A (1989), 149-158.

[5] M. Escobedo and L. Vega, A semilinear Dirac equation in $H^{s}\left(\mathbf{R}^{\mathbf{3}}\right)$ for $s>1$, SIAM J. Math. Anal., 2 (1997), 338-362.

[6] M. Keel and T. Tao, Endpoint Strichartz estimates, Amer. J. Math., 120 (1998), 955980.

[7] S. Machihara, K. Nakanishi and T. Ozawa, Small global solutions and the nonrelativistic limit for the nonlinear Dirac equation, Rev. Mat. Iberoamericana 19 (2002), 179-194.

[8] W. A. Strauss, Nonlinear invariant wave equations, in Invariant wave equations, vol. 73 of Lecture Notes in Phys., Springer, Berlin, 1978, 197-249. 